Supporting Information

\title{
Ionic Liquid-Assisted Electropolymerization for \\ Lithographical Perfluorocarbon Deposition and Hydrophobic Patterning
}

Jhih-Guang Wu, ${ }^{\dagger, \uparrow}$ Cheng-Yang Lee, ${ }^{\star, " ~ S h a o-S h u o ~ W u, ~ a n d ~ S h y h-C h y a n g ~ L u o ~}{ }^{* \dagger}$

${ }^{\dagger}$ Department of Materials Science and Engineering, College of Engineering, National Taiwan University, No. 1, Sec. 4, Roosevelt Road, Taipei, 10617 Taiwan

Department of Materials Science and Engineering, National Cheng Kung University, 1 University Road, Tainan 70101, Taiwan.

Email: shyhchyang@ntu.edu.tw 

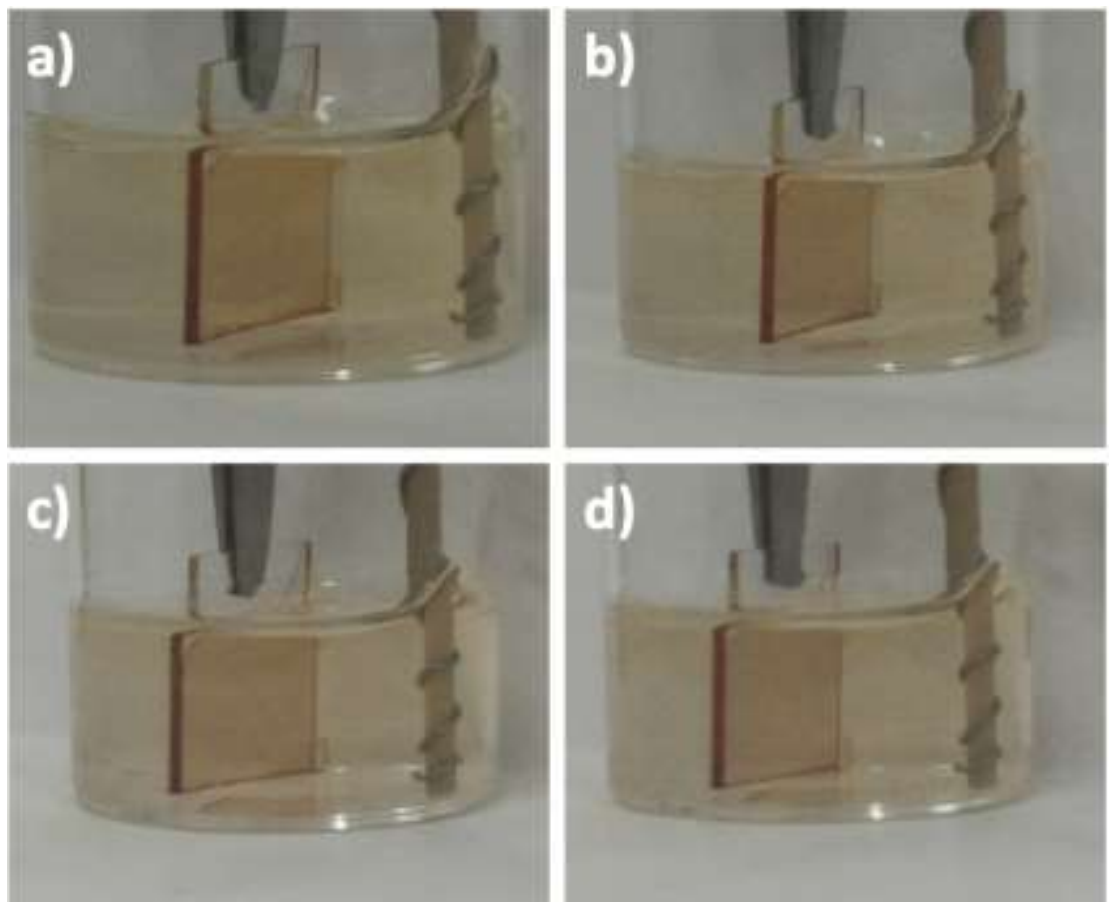

Figure S1. The stability test of photoresists in ILs. a) the ITO with photoresists immersed in ILs; b) after 10 mins; c) after applying a constant voltage of $1.0 \mathrm{~V} \mathrm{vs} \mathrm{Ag} / \mathrm{AgCl}$; d) after applying a constant voltage of $-1.0 \mathrm{~V}$ vs $\mathrm{Ag} / \mathrm{AgCl}$. 

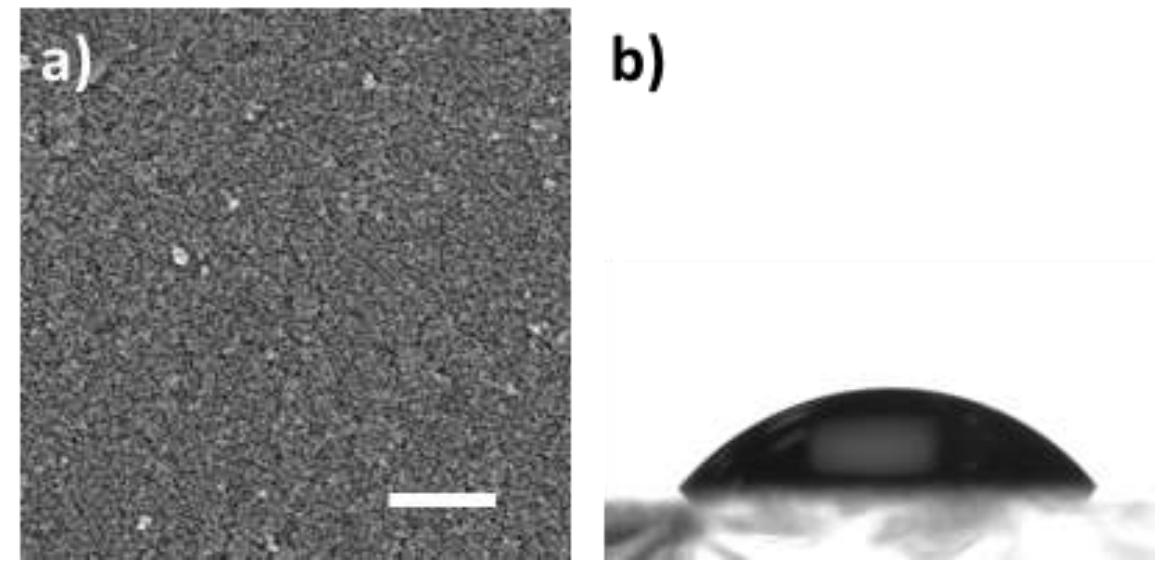

Figure S2. a) The SEM image of poly(EDOT-OH) and (b) the water contact angle result showing a hydrophilic surface. The scale bar represents $500 \mathrm{~nm}$. 

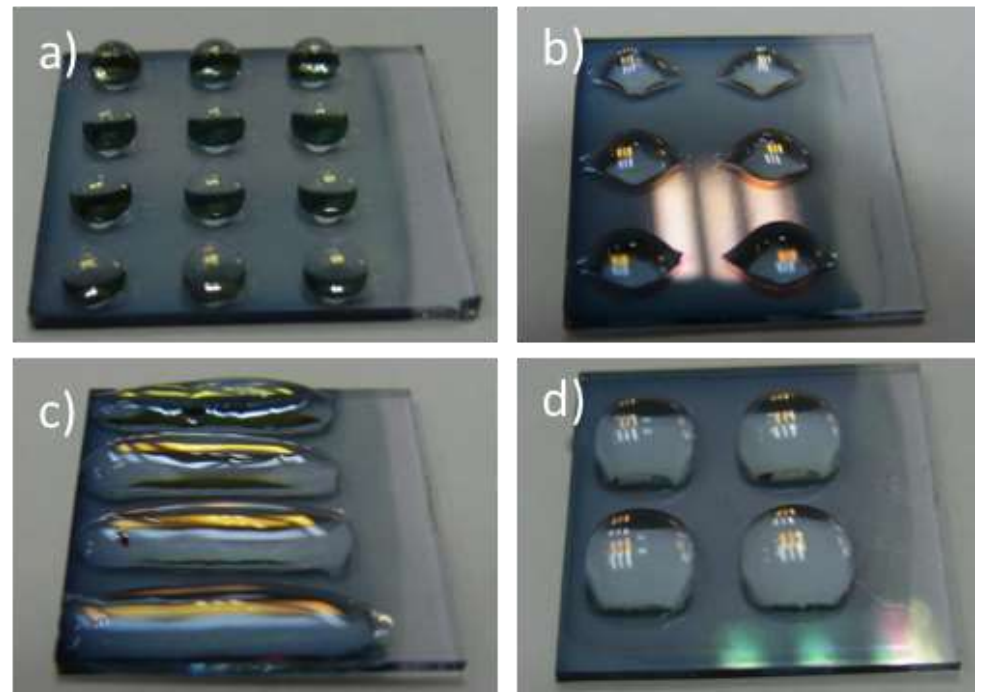

Figure S3. a) Circle shape pattern on ITO substrate; b) diamond shape pattern on ITO substrate; c) Rectangular shape pattern on ITO substrate; d) square shape pattern on ITO substrate. 

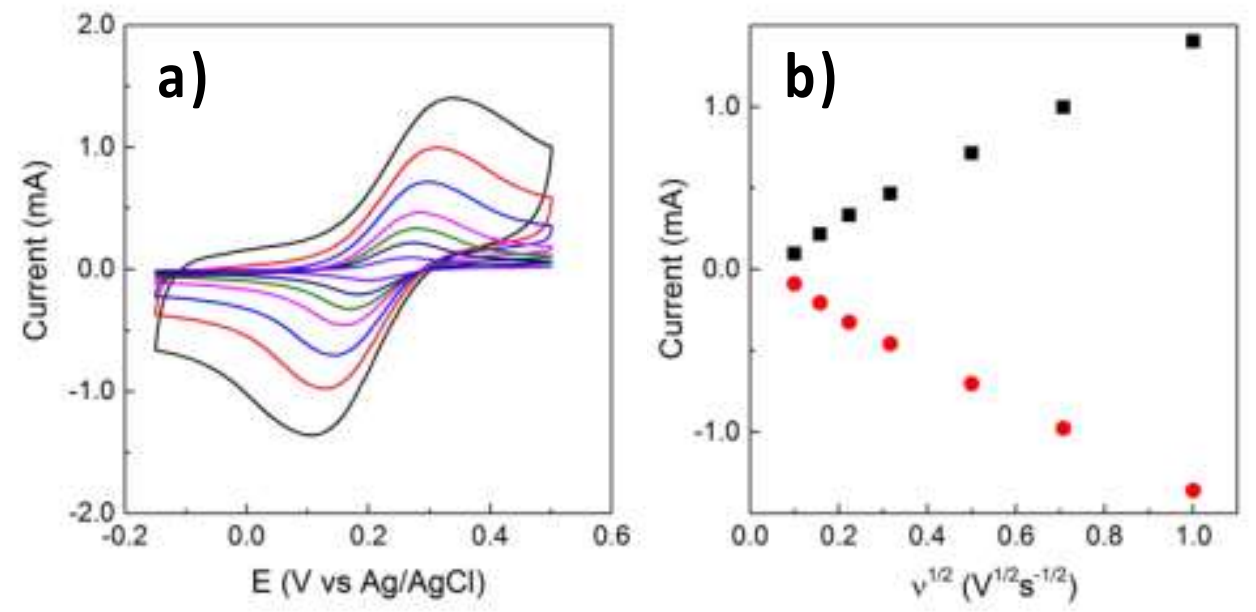

Figure S4. a) The cyclic voltammogram showing the redox behavior of $\mathrm{Fe}^{2+} / \mathrm{Fe}^{3+}$ at scan rates of 10 (purple), 20 (navy), 50 (green), 100 (pink), 200 (blue), 500 (red), and 1000 (black) mV s-1, b) Anodic (black) and cathodic (red) peak current vs. square root of scan rate. 